\title{
Additive effects of physical exercise and environmental enrichment on adult hippocampal neurogenesis in mice
}

\author{
Klaus Fabel 1,2,3, Susanne A. Wolf2,3,4, Dan Ehninger ${ }^{2,3}$, Harish Babu ${ }^{2,3}$, Perla Leal-Galicia' \\ and Gerd Kempermann ${ }^{1,2,3}$ * \\ CRTD - DFG Research Center for Regenerative Therapies, Dresden, Germany \\ 2 Max Delbrück Center for Molecular Medicine Berlin-Buch, Berlin, Germany \\ ${ }^{3}$ Volkswagenstiftung Research Group, Department of Experimental Neurology, Charité University Medicine Berlin, Berlin, Germany \\ 4 Institute of Anatomy, Department of Cell and Neurobiology, University Zürich, Zürich, Switzerland
}

\section{Edited by:}

Jeffrey D. Macklis, Massachusetts General Hospital, USA

\section{Reviewed by:}

Ronald S. Duman, Yale University

School of Medicine, USA

Daniel A. Peterson, Rosalind Franklin

University of Medicine and Science, USA

Francis G. Szele, Oxford University, UK

\section{*Correspondence:}

Gerd Kempermann, CRTD - DFG

Research Center for Regenerative

Therapies Dresden, Tatzberg 47-49,

01307 Dresden, Germany.

e-mail: gerd.kempermann@crtdresden.de
Voluntary physical exercise (wheel running, $R \cup N$ ) and environmental enrichment both stimulate adult hippocampal neurogenesis but do so by different mechanisms. RUN induces precursor cell proliferation, whereas ENR exerts a survival-promoting effect on newborn cells. In addition, continued RUN prevented the physiologically occurring age-related decline in precursor cell in the dentate gyrus but did not lead to a corresponding increase in net neurogenesis. We hypothesized that in the absence of appropriate cognitive stimuli the potential for neurogenesis could not be realized but that an increased potential by proliferating precursor cells due to RUN could actually lead to more adult neurogenesis if an appropriate survival-promoting stimulus follows the exercise. We thus asked whether a sequential combination of RUN and ENR (RUNENR) would show additive effects that are distinct from the application of either paradigm alone. We found that the effects of 10 days of RUN followed by 35 days of ENR were additive in that the combined stimulation yielded an approximately $30 \%$ greater increase in new neurons than either stimulus alone, which also increased neurogenesis. Surprisingly, this result indicates that although overall the amount of proliferating cells in the dentate gyrus is poorly predictive of net adult neurogenesis, an increased neurogenic potential nevertheless provides the basis for a greater efficiency of the same survival-promoting stimulus. We thus propose that physical activity can "prime" the neurogenic region of the dentate gyrus for increased neurogenesis in the case the animal is exposed to an additional cognitive stimulus, here represented by the enrichment paradigm.

Keywords: stem cell, hippocampus, reserve, learning

\section{INTRODUCTION}

In the adult hippocampus, new granule cell neurons develop from a resident population of radial glia-like precursor cells over a series of identifiable intermediate steps (Kempermann et al., 2004). In the course of neuronal development different stages are differentially regulated and influenced by inherited traits and differentially sensitive to external stimuli. We are particularly interested in how "activity" regulates adult neurogenesis and broadly distinguish between the effects of physical exercise and cognitive stimulation. Physical exercise, like voluntary running in a running wheel (RUN), increases the proliferation of precursor cells in the subgranular zone of the dentate gyrus, thereby expanding the pool of progenitor cells that are available for further differentiation (van Praag et al., 1999; Kronenberg et al., 2003). In contrast, stimuli that are supposedly more specific to hippocampal function have no or limited effect on cell proliferation but recruit new neurons for long-term survival. We have used environmental enrichment (ENR) to demonstrate this survival-promoting effect (Kempermann et al., 1997, 2002; Kempermann and Gage, 1999; Kronenberg et al., 2003); others have shown similar results with specific stimuli such as learning tasks (Gould et al., 1999; Dobrossy et al., 2003; Leuner et al., 2004; Hairston et al., 2005). For a more detailed review of this difference and possible underlying mechanisms see (Olson et al., 2006; Fabel and Kempermann, 2008). Based on a population genetic study we found that most of the net neurogenic regulation is determined not by the expansion phase but rather by the control of survival (Kempermann et al., 2006), which might indicate that a survivalpromoting effect in response to a cognitive stimulus exerts greater definite control on adult neurogenesis than the activity-dependent expansion of the precursor cell pool. Finally, we found that continued physical activity counteracted the physiological decrease in precursor cell proliferation in the aging dentate gyrus and thereby maintained the potential for neurogenesis at a level corresponding to a much younger age (Kronenberg et al., 2006). Supposedly in the absence of additional cognitive stimuli this potential, however, was not translated into a net increase in neurogenesis.

At this point the obvious question seemed, that if precursor cell proliferation were enhanced by RUN, would subsequent exposure to ENR recruit additional new neurons from the increased pool of progenitor cells (RUNENR)? In that case, the increase due to the sequential stimulation might theoretically be either proportional or over-proportional compared to the effects of enrichment without prior priming by physical activity. Alternatively, if the recruitment by ENR were independent of the size of the available precursor 
cell pool - as long as this pool was large enough to allow adequate recruitment - we expect to find net neurogenesis in RUNENR identical to enrichment alone. In that case, only the functional demand would dictate the magnitude of the neurogenic effect.

From our previous experiments we knew that ENR would not stimulate proliferation (Steiner et al., 2008) and that upon sustained exposure to RUN the acute effects of RUN on precursor cell proliferation would wear off (Kronenberg et al., 2003). The peak of the pro-proliferative effect (in terms of the number of precursor cells in cell cycle) had been after 10 days, so that we chose 10 days for the duration of the RUN phase. We chose the following ENR phase to be 5 weeks because after such period new granule cells have matured and numbers have stabilized (van Praag et al., 2002; Jessberger and Kempermann, 2003; Kempermann et al., 2003). Finally, the ENR phase began only after the cells had been labeled with bromodeoxyuridine (BrdU) during the last 3 days of the RUN phase to ensure that we would only measure the ENR-induced survival effect on a population of cells that had previously responded to the RUN stimulus. The experimental design is depicted in Figure 1.

\section{MATERIALS AND METHODS ANIMALS AND EXPERIMENTAL DESIGN}

Forty female C57BL/6 mice, 8 -weeks old at the beginning of the experiment, were obtained from Charles River (Sulzfeld, Germany). The mice were randomly distributed to four experimental groups, $N=10$ per group (Figure 1). All animals were kept in the same room with a constant 12-h-light/dark-cycle and were fed with the same food and water ad libitum. All applicable local and federal regulations of animal welfare were followed. We used female mice because enriched environment experiments with male mice are confounded by their territorial behavior and the development of a strong and potentially stressful social hierarchy. All of our previous studies, to which we relate, have been done in female animals as well.

The experimental period lasted 45 days, divided into a first phase of 10 days and a second phase of 35 days (Figure 1). According to our hypothesis the main experimental group consisted of mice that lived in a cage equipped with a running wheel during the first phase, followed by the exposure to an enriched environment in the second (RUNENR). The idea was that in this condition, running would prime the hippocampus for the effects of enrichment that followed. Consequently, the first control condition consisted of mice that were running in the first phase but lived under control conditions in the second (RUNSTD). Because all animals were analyzed at the end of the 45 days, the measured effects of running in this condition represented sustained effects after 35 days of discontinuation of physical exercise. RUNSTD and RUNENR differed in the second phase only, not the first. The effects of ENR alone were assessed in a group that lived under control conditions in the first period but under enrichment conditions in the second (STDENR). In relation to RUNENR, the STDENR thus differed only in the first phase, not the second. All three groups were compared to mice living under control conditions (STDSTD). At the end of the first period, five mice of each group received single daily injections of persistent S-phase label bromodeoxyuridine (BrdU; $50 \mu \mathrm{g} / \mathrm{g}$ body weight in $0.9 \%$ saline; Sigma) for 3 days. The putative survival-promoting effect of the ENR stimulus in phase 2 would thereby act on a cohort of progenitor cells generated in the last 3 days of phase 1 . The remaining animals were intended for a gene expression study, which failed technically.

\section{TISSUE PREPARATION}

The five animals from each group that had been injected with BrdU were deeply anesthesized with ketamine and xylazine and perfused with $0.9 \% \mathrm{NaCl}$ solution followed by $4 \%$ paraformaldehyde in $0.1 \mathrm{M}$ phosphate buffered saline. The brains were removed and fixed in $4 \%$ paraformaldehyde for $24 \mathrm{~h}$ and then transferred in $30 \%$ sucrose. For the generation of coronal section series $(40 \mu \mathrm{m})$, brains were mounted on a dry-ice cooled copper block that was attached to a sliding microtome (Leica). Sections were stored in a cryoprotectant solution (25\% ethylene glycol, $25 \%$ glycerol and $50 \% 0.1 \mathrm{M}$ phosphate buffer; $\mathrm{v} / \mathrm{v}$ ) at $-20^{\circ} \mathrm{C}$.

The sections were stained using free-floating immunohistochemistry and prepared for BrdU detection by incubation in $2 \mathrm{~N}$ $\mathrm{HCl}$ for $30 \mathrm{~min}$ at $37^{\circ} \mathrm{C}$ and subsequent washing in $0.1 \mathrm{M}$ borate buffer ( $\mathrm{pH} 8.5$ ) for $10 \mathrm{~min}$.

\section{ANTIBODIES}

All antibodies were diluted in Tris-buffered saline (TBS; $\mathrm{pH}=8.0$ ) containing $0.2 \%$ Triton X-100 and 3\% donkey serum. We here used the following primary antibodies: rat anti-BrdU (Harlan Seralab) 1:500, mouse anti-NeuN (Chemicon) 1:100, goat antiDcx (Santa Cruz) 1: 250, rabbit anti-S100 $\beta$ (Swant) 1:2000. Secondary antibodies, raised in donkey and conjugated with either FITC, RhodamineX, CY-5 or Biotin were purchased from Jackson Laboratories (Distributor: Dianova) and diluted 1:500 for immunohistochemistry.

\section{IMMUNOHISTOCHEMISTRY AND MICROSCOPY}

For quantification of BrdU-immunoreactive cells, every sixth section of the coronal section series was subjected to immunohistochemistry. Here, primary antibodies were recognized with biotinylated secondary antibodies and visualized with the peroxidase method (ABC system, Vectastain ${ }^{\circledR}$, Vector Laboratories) and nickel-intensified diaminobenzidine as chromogen. Using conventional light microscopy, BrdU-immunoreactive cells in the subgranular zone and granule cell layer of the dentate gyrus were counted throughout the entire rostro-caudal extent of the hippocampus. The optical dissector method was modified in that cells appearing in the uppermost focal plane were excluded from analysis, as described previously (Kempermann et al., 2003).

For phenotyping of BrdU-immunoreactive cells, every twelfth section of the coronal section series was subjected to tripleimmunefluorescence labeling. After pretreatment for BrdU detection (see above) and a blocking step with Tris-buffered saline-plus (TBS-plus, containing 3\% donkey serum and $0.2 \%$ Triton $\mathrm{X}-100$ ) sections were incubated in the respective primary antibody at $4^{\circ} \mathrm{C}$ for $48 \mathrm{~h}$. After washing sections in TBS and TBS-plus, we exposed them to the respective fluorochrome-conjugated secondary antibodies for $4 \mathrm{~h}$ at room temperature. Labeled sections were mounted in polyvinyl alcohol with diazabicyclo-octane as antifading substance.

BrdU-positive cells were phenotypically characterized in triplefluorescent labeled sections using confocal laser scanning microscopy (Leica TCS SP2). All confocal analyses were conducted in 
sequential scanning mode to avoid cross-bleeding between channels and series of images along the $z$-axis ( 1 optical section/ $1 \mu \mathrm{m}$ ) of BrdU-positive cells were taken to demonstrate colocalization of immunoreactivity against BrdU and the respective antigen in the same cell. One hundred BrdU-positive cells randomly chosen from the subgranular zone and granule cell layer of the dentate gyrus were examined for NeuN or S100 $\beta$ immunoreactivity. Percentages of immunohistochemical phenotypes were multiplied with the total number of BrdU-immunoreactive cells to achieve the absolute number of the respective phenotype.

\section{STATISTICAL ANALYSES}

All statistical analyses of morphological data were done with Statview 4.5.1 for Macintosh. Factorial analyses of variance (ANOVA) were used, followed by Fisher post hoc test, where appropriate.

\section{RESULTS}

In view of previous studies (van Praag et al., 1999; Kronenberg et al., 2003), we predicted that both STDENR and RUNSTD would increase adult neurogenesis as assessed by the number of BrdUlabeled cells that expressed neuronal marker NeuN 5 weeks after the injection of BrdU. The important difference to the previous reports was the temporal separation of the two stimuli, RUN in phase 1 and ENR in phase 2 (Figure 1) combined with STD conditions for the remaining time. The pro-proliferative stimulus of RUN and the survival-promoting stimulus of ENR (Kronenberg et al., 2003) were deliberately directed to the phase of their largest presumed effectiveness in relation to the time-point of precursor cell division.

All animals were injected with BrdU in the last 3 days of the 1 st phase of the experiment, hence before the 2 nd phase of the experiment (Figure 1). We first assessed the total number of BrdUpositive cells per hippocampus (Figures 2A,B,E-H and 3, 1st group of bars; ANOVA: $\left.F_{(3,16)}=9.34, p=0.0008\right)$. The direct comparison of RUNSTD against STDSTD revealed that RUNSTD alone had an increasing effect on net neurogenesis, although the exercise had been discontinued during the postmitotic period, in which any survival-promoting effect would have to be expected. This result was visible on the level of both the number of BrdU-labeled cells (Figures 2E,G and 3, 1st group of bars), reflecting the survival of newborn cells, as well as the number of neurons (BrdU/NeuN) among these cells (Figures 2C,D and 3, 2nd group of bars; ANOVA: $\left.F_{(3,16)}=11.49, p=0.0003\right)$. From this finding we can conclude that RUN seems to induce an intrinsic program of neurogenesis that can evolve even in the absence of sustained activity. Consistent with previous data STDENR also appeared to increase adult neurogenesis compared to STDSTD but the direct comparison here missed conventional statistical significance ( $p=0.06$ for BrdU/NeuN and $p=0.08$ for total survival). There was no difference between RUNSTD and STDENR for either of these parameters.

The sequential combination of RUN and ENR led to significantly greater BrdU-counts compared to RUNSTD and to STDENR (as well as to STDSTD). This was also reflected in the number of BrdU/NeuN-double positive cells (Figures 2D and 3, 2nd group of bars). STDENR and RUNENR caused an increase in astrocytes (as measured by BrdU/S-100ß-positive cells, Figure 3, $3^{\text {rd }}$ group of bars; ANOVA: $\left.F_{(3,16)}=3.89, p=0.03\right)$. BrdU-cells of an undetermined phenotype were significantly increased in STDENR and RUNENR as well (Figure 3, 4th group of bars; ANOVA: $\left.F_{(3,16)}=3.89, p=0.03\right)$.

We calculated the relative effects of enrichment on either naïve mice (STDSTD) or mice primed with physical exercise (RUNSTD) and found an increase by factor 1.32 for the comparison STDENR against STDSTD and 1.38 (based on the means) for the comparison RUNENR vs. RUNSTD. We conclude that the effects of enrichment on neurogenesis are thus dependent on the potential provided by proliferative precursor cells.

\section{DISCUSSION}

Here we have shown that, as hypothesized from a previous study (Kronenberg et al., 2006), physical activity can "prime" the hippocampal dentate gyrus towards an increased level of neurogenesis elicited by ENR. In contrast to previous enrichment experiments in our group (Kronenberg et al., 2003), exposure to the enriched

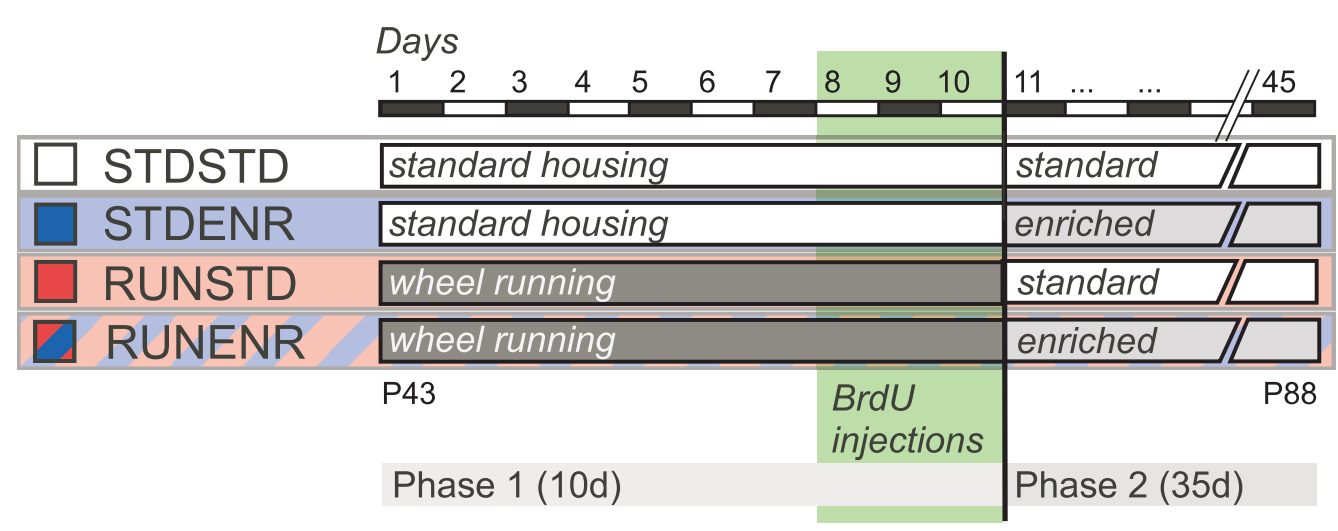

FIGURE 1 | Experimental design. During phase 1 of the experiment mice were either given access to a RUN ad libitum (RUNSTD and RUNENR group) or were housed under standard laboratory conditions (STDSTD and STDENR group). Dividing hippocampal precursor cells were labeled with BrdU during the last 3 days of phase 1. Subsequently, in phase 2 (duration: 35 days), animals were either exposed to an enriched environment (STDENR and RUNENR groups) or housed under standard laboratory conditions (STDSTD and RUNSTD group). Animals were killed after phase 2 was completed and brain tissue was prepared for immunohistochemistry. For further details see Sections "Introduction and Materials and Methods". 

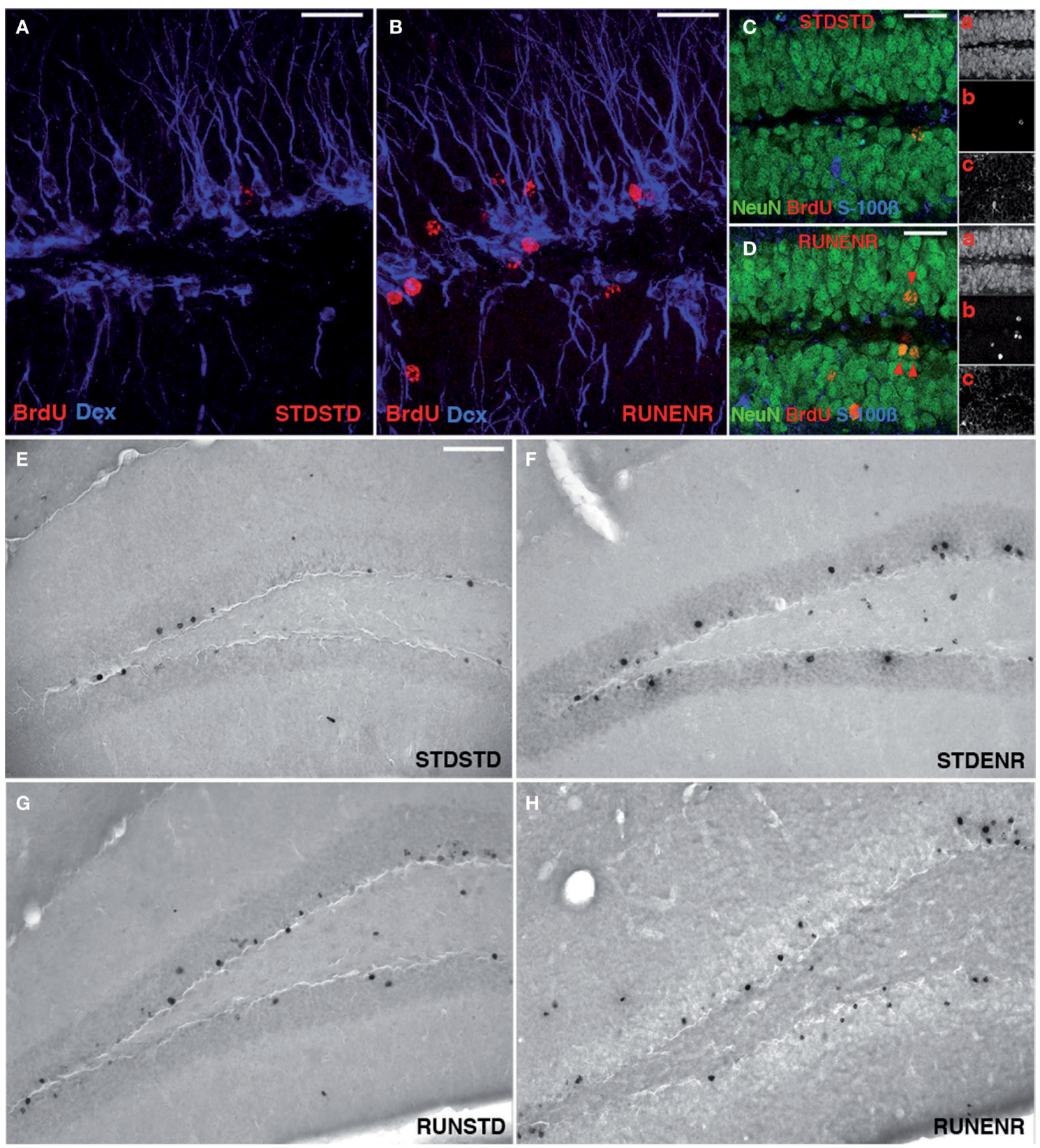

FIGURE 2 |Activity-induced changes in the number of BrdU-labeled cells and new neurons in the dentate gyrus. $(A, B)$ Confocal projections of the dentate gyrus (z-stack of 10 optical sections with 1.7- $\mu$ m thickness). BrdU, Red; DCX, Blue. Scale bar, $25 \mu \mathrm{m}$ in (A-D) Confocal image (optical section of 1- $\mu \mathrm{m}$ thickness) of
STDSTD in (C) and RUNENR in (D) showing NeuN, Green; BrdU, Red, S100ß, Blue; Inset: $a, \mathrm{NeuN}$; b, BrdU; c, S100ß. (D) Arrowheads in red indicating colocalization of BrdU and NeuN. Scale bar, $25 \mu \mathrm{m}$ in (C,D). (E-H) Brightfield images of BrdUpositive cells in the dentate gyrus. Scale bar (in $\mathbf{E}$ for $\mathbf{E}-\mathbf{H}$ ), $100 \mu \mathrm{m}$. environment in ENR began only after labeling dividing precursor cells with BrdU. Consequently, data for ENR reveal only effects of enrichment on cells in a postmitotic stage. The results of an increased number of new neurons (BrdU/NeuN positive) confirm that enrichment exerts a survival-promoting effect on the progeny of dividing cells.

Taken together these data indicated that our experimental paradigm showed the expected effects with regards to both RUN and ENR, albeit with an important modification over previous studies. Analysis of the RUNENR condition revealed an even larger effect on adult neurogenesis than either stimulus alone (Figure 3). This finding suggests that effects on precursor cell proliferation (as in RUNSTD) and survival of the progeny (as in STDENR) are additive.

This finding further supports our hypothesis that physical exercise might help to maintain a potential for adult neurogenesis that goes unused in the absence of appropriate additional (cognitive) stimuli (Kempermann, 2008). Environmental enrichment as 


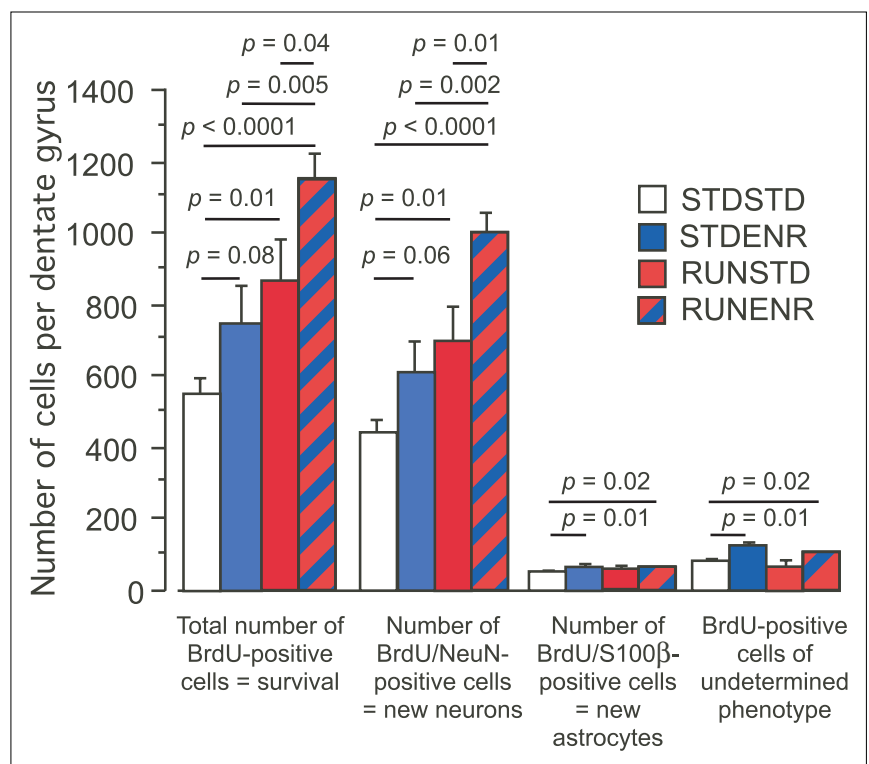

FIGURE 3 |The number of BrdU-labeled cells in the dentate gyrus was determined along with the cellular phenotypes of BrdU-positive cells (NeuN indicating a neuronal, S100 $\beta$ an astroglial fate). We found RUNSTD to cause an increased number of BrdU-labeled cells as well as new neurons compared to STDSTD. RUNENR resulted in a further increase in the number of BrdU-positive cells and newborn neurons. Therefore, the effects of wheel running and environmental enrichment on adult hippocampal neurogenesis were additive. Fisher post hoc test after ANOVA.

well as (at least in some studies) specific learning stimuli exert a survival-promoting effect on newborn neurons, whereas physical activity acts primarily through the proliferation of precursor cells (Kronenberg et al., 2003).

Precursor cells, arguably, represent the cellular potential for neurogenesis. Selection for survival largely occurs on the postmitotic stage, when the cells have made first synaptic contacts (Brandt et al., 2003; Kempermann et al., 2004). Enrichment during a critical period in the development of a new cell would serve as an appropriate behavioral stimulus to exert this rescuing function. The present experiment was designed to maximize this effect. With a period of 10 days of exercise we chose a paradigm that would lead to a maximum of new dividing cells, as demonstrated previously (Kronenberg et al., 2006). With 5 weeks of enrichment we safely covered the window of activity-dependent selection assumed by most authors (Gould et al., 1999; Greenough et al., 1999; Dobrossy et al., 2003; Leuner et al., 2004; Hairston et al., 2005; Ehninger and Kempermann, 2006). It should be noted that in long-term experiments, both ENR and voluntary wheel running have effects that are not readily visible in acute settings, because an additional survival-promoting effect acts upon the precursor cells and not just their progeny in both paradigms (Kempermann and Gage, 1999; Kempermann et al., 2002; Kronenberg et al., 2006). Long-term wheel running had both pro-proliferative and survivalpromoting effects, the latter effect being responsible for the increase in "potential" for neurogenesis (Kronenberg et al., 2006). When we published our first report on the effects of exposure to an enriched environment on adult hippocampal neurogenesis in mice in 1997 (Kempermann et al., 1997), our enriched environments contained running wheels because we had assumed that physical activity would have to be part of any enrichment that deserved this name (Rosenzweig and Bennett, 1996). We later found that physical exercise alone had distinct effects on adult hippocampal neurogenesis (van Praag et al., 1999). In all our studies after 1998, the enriched environments did not contain running wheels. The present morphological and molecular data confirm that both "activity" paradigms are substantially different and can be combined to obtain specific additive regulatory effects.

Our present data also support the idea (at $p=0.06$ ) that ENR exerts a survival-promoting neurogenic effect in the absence of a preconditioning of the progenitor cell population by a proproliferative stimulus. In a previous study we had exposed mice to ENR immediately after injecting a single dose of BrdU and examined the brains $24 \mathrm{~h}$ later. In that study we had found that ENR had an acute effect on the late, neuronally committed intermediate progenitor cells (type- 3 cells) and not only on the postmitotic progeny (Steiner et al., 2008). In the ENR groups of the present study the animals were also placed in the enriched environment after BrdU had been injected. The number of cells of an undetermined phenotype was significantly increased in the STDENR group, consistent with an effect on precursor cells and not only net neurogenesis. Long-term stimulation by ENR resulted in a lasting increase in proliferating precursor cells (Kempermann et al., 2002). A similar increase persisted also after withdrawal from ENR for 3 months (Kempermann and Gage, 1999). A follow-up study will now address the changes at the level of the precursor cells in RUNENR compared to STDENR and RUNSTD.

It has sometimes been postulated that the particular type of physical activity might influence the results, because in studies on the effects of water maze training on neurogenesis, a yoked control that swam for the same time as the groups in the learning test had no signs of increased neurogenesis (van Praag et al., 1999; Ehninger and Kempermann, 2006). However, in those studies swimming lasted only a few minutes per day, so that from those data the conclusion that swimming would be less efficient than running is not justified (Ra et al., 2002). Voluntary wheel running and forced exercise in treadmill paradigms thus remain the beststudied paradigms of physical exercise in rodents with respect to their effects on adult neurogenesis (van Praag et al., 1999, 2005; Trejo et al., 2001; Ra et al., 2002; Kronenberg et al., 2003, 2006; Kim et al., 2004; Naylor et al., 2005; Wolf et al., 2006; Fabel and Kempermann, 2008). The focus on running is problematic because physical activity is more than those aspects assessable in the wheel running paradigm. In addition, other modalities of activity might provide equivalent stimulation upon investigation.

In theory, also a completely reverse experiment is imaginable, in which the effects of RUN could be investigated in animals that had previously experienced an enriched environment. In terms of the order, in which stimuli act in the course of neuronal development (first stimulation of proliferation than selective induction of survival) the focus on the sequence RUNENR (rather than ENRRUN) seemed logical. From our previous and the new data no simple hypothesis could be derived what to expect under the reverse experimental conditions. On the other hand, stress has been shown to interfere with the exercise-induced regulation of 
adult neurogenesis (Stranahan et al., 2006; Kannangara et al., 2009), so that also beneficial modulation through ENR is conceivable. In contrast to the relatively acute situation in the present study, we know from long-term experiments (over 6 months) that ENR can increase the pool of precursor cells (Kempermann and Gage, 1999; Kempermann et al., 2002) similar to how does exercise (Kronenberg et al., 2006). A considerably different experimental design than in the present study would be required to investigate a potential interaction between RUN and ENR in that long-term setting.

A surprising key finding of the present study was that the relative increase in neurogenesis evoked by ENR was the same (about plus 30\%) in ENR vs. CTR and in RUNENR vs. RUN, suggesting that very limited interaction effects existed. This would imply that the size of the population of proliferating precursor cells would affect the number of cells that are recruited by enrichment. Alternatively one might have expected that, if a given cognitive stimulus recruited only exactly the number of neurons that stood in some inherent relation to the stimulus itself, further increasing the number of proliferating precursor cells would not increase the number of new neurons. Thus, although precursor cell proliferation only determines roughly $20 \%$ of the variation found in net neurogenesis on a genetic level (Kempermann et al., 2006), the number of precursor cells being "activated" by running affected

\section{REFERENCES}

Brandt, M. D., Jessberger, S., Steiner, B., Kronenberg, G., Reuter, K., BickSander, A., von der Behrens, W., and Kempermann, G. (2003). Transient calretinin expression defines early postmitotic step of neuronal differentiation in adult hippocampal neurogenesis of mice. Mol. Cell. Neurosci. 24, 603-613.

Dobrossy, M.D., Drapeau, E.,Aurousseau, C., Le Moal, M., Piazza, P. V., and Abrous, D. N. (2003). Differential effects of learning on neurogenesis: learning increases or decreases the number of newly born cells depending on their birth date. Mol. Psychiatry 8, 974-982.

Ehninger, D., and Kempermann, G. (2006). Paradoxical effects of learning the Morris water maze on adult hippocampal neurogenesis in mice may be explained by a combination of stress and physical activity. Genes Brain Behav. 5, 29-39.

Fabel, K., and Kempermann, G. (2008). Physical activity and the regulation of neurogenesis in the adult and aging brain. Neuromolecular Med. 10, 59-66.

Gould, E., Beylin, A., Tanapat, P., Reeves, A., and Shors, T. J. (1999). Learning enhances adult neurogenesis in the hippocampal formation. Nat. Neurosci. 2, 260-265.

Greenough, W. T., Cohen, N. J., and Juraska, J. M. (1999). New neurons in old brains: learning to survive? Nat Neurosci. 2, 203-205.

Hairston, I. S., Little, M. T., Scanlon, M. D., Barakat, M. T., Palmer, T. D., Sapolsky, R. M., and Heller, H. C. (2005). Sleep restriction suppresses neurogenesis induced by hippocampusdependent learning. J. Neurophysiol. 94, 4224-4233.

Jessberger, S., and Kempermann, G. (2003).Adult-born hippocampal neurons mature into activity-dependent responsiveness. Eur. J. Neurosci. 18, 2707-2712.

Kannangara, T. S., Webber, A., GilMohapel, J., and Christie, B. R. (2009). Stress differentially regulates the effects of voluntary exercise on cell proliferation in the dentate gyrus of mice. Hippocampus 19, 889-897.

Kempermann, G. (2008). The neurogenic reserve hypothesis: what is adult hippocampal neurogenesis good for? Trends Neurosci. 31, 163-169.

Kempermann, G., Chesler, E. J., Lu, L., Williams, R. W., and Gage, F.H. (2006). Natural variation and genetic covariance in adult hippocampal neurogenesis. Proc. Natl. Acad. Sci. U.S.A. 103 780-785.

Kempermann, G., and Gage, F. H. (1999). Experience-dependent regulation of adult hippocampal neurogenesis: effects of long-term stimulation and stimulus withdrawal. Hippocampus 9, 321-332.

Kempermann, G., Gast,D., and Gage, F. H. (2002). Neuroplasticity in old age: the net effect on neurogenesis. It is thus conceivable that physical exercise increases neurogenic potential beyond the proliferation of precursor cells.

The comparable relative increase in neurogenesis by about $30 \%$, independent of previous exposure to the RUN condition does not prove that the effect is in fact identical because we do not know the baseline values at 10 days. With those data at hand one could also ask whether under RUNENR conditions the relative survivalpromoting effect of ENR in relation to the number of (labeled) cells available after 10 days might be different after previous "priming" with RUN. Future studies will address this possibility.

In summary, our data indicate that physical activity and exposure to an enriched environment act through different mechanisms but are additive in their effect on adult hippocampal neurogenesis. We propose that physical activity can "prime" the neurogenic region of the dentate gyrus for increased neurogenesis, if exposed to an identical more cognitive stimulus as presumably represented by the enrichment paradigm.

\section{ACKNOWLEDGMENTS}

This study was supported by Volkswagenstiftung. We thank Josefine Ruß and Ruth Zarmstorff for excellent technical assistance and Rupert Overall for helpful discussions. P. Leal-Galicia was supported by CONACyT, México and DGAPA 181550 and DGP, UNAM. sustained fivefold induction of hippocampal neurogenesis by long-term environmental enrichment. Ann. Neurol. 52, 135-143.

Kempermann,G., Gast,D., Kronenberg, G., Yamaguchi, M., and Gage, F.H. (2003) Early determination and long-term persistence of adult-generated new neurons in the hippocampus of mice. Development 130, 391-399.

Kempermann, G., Jessberger, S. Steiner, B., and Kronenberg, G. (2004). Milestones of neuronal development in the adult hippocampus. Trends Neurosci. 27, 447-452.

Kempermann, G., Kuhn, H. G., and Gage, F. H. (1997). More hippocampal neurons in adult mice living in an enriched environment. Nature 386, 493-495.

Kim, Y. P., Kim, H., Shin, M. S., Chang, H. K., Jang, M. H., Shin, M. C., Lee, S J., Lee, H. H., Yoon, J. H., Jeong, I. G., and Kim, C. J. (2004). Age-dependence of the effect of treadmill exercise on cell proliferation in the dentate gyrus of rats. Neurosci. Lett. 355, 152-154.

Kronenberg, G., Bick-Sander, A. Bunk, E., Wolf, C., Ehninger, D., and Kempermann, G. (2006). Physical exercise prevents age-related decline in precursor cell activity in the mouse dentate gyrus. Neurobiol. Aging 27, 1505-1513.

Kronenberg, G., Reuter, K., Steiner, B., Brandt,M.D., Jessberger,S., Yamaguchi, M., and Kempermann, G. (2003). Subpopulations of proliferating cells of the adult hippocampus respond differently to physiologic neurogenic stimuli. J. Comp. Neurol. 467, 455-463.

Leuner, B., Mendolia-Loffredo, S., Kozorovitskiy, Y., Samburg, D., Gould, E., and Shors, T. J. (2004). Learning enhances the survival of new neurons beyond the time when the hippocampus is required for memory. J. Neurosci. 24, 7477-7481.

Naylor, A. S., Persson, A. I., Eriksson, P. S., Jonsdottir, I. H., and Thorlin, T. (2005). Extended voluntary running inhibits exercise-induced adult hippocampal progenitor proliferation in the spontaneously hypertensive rat. J. Neurophysiol. 93, 2406-2414.

Olson, A. K., Eadie, B. D., Ernst, C., and Christie, B. R. (2006). Environmental enrichment and voluntary exercise massively increase neurogenesis in the adult hippocampus via dissociable pathways. Hippocampus 16, 250-260.

Ra, S. M., Kim, H., Jang, M. H., Shin, M. C., Lee, T. H., Lim, B. V., Kim, C. J., Kim, E. H., Kim, K. M., and Kim, S. S. (2002). Treadmill running and swimming increase cell proliferation in the hippocampal dentate gyrus of rats. Neurosci. Lett. 333, 123-126.

Rosenzweig, M. R., and Bennett, E. L. (1996). Psychobiology of plasticity: effects of training and experience on brain and behavior. Behav. Brain Res. 78, 57-65. 
Steiner, B., Zurborg, S., Horster, H., Fabel, K., and Kempermann, G. (2008). Differential $24 \mathrm{~h}$ responsiveness of Prox1-expressing precursor cells in adult hippocampal neurogenesis to physical activity, environmental enrichment, and kainic acid-induced seizures. Neuroscience 154, 521-529.

Stranahan,A.M., Khalil, D., and Gould,E. (2006). Social isolation delays the positive effects of running on adult neurogenesis. Nat. Neurosci. 9, 526-533.

Trejo, J.L., Carro, E., and Torres-Aleman, I. (2001). Circulating insulin-like growth factor I mediates exercise-induced increases in the number of new neurons in the adult hippocampus. J. Neurosci. 21, 1628-1634. van Praag, H., Kempermann, G., and Gage, F. H. (1999). Running increases cell proliferation and neurogenesis in the adult mouse dentate gyrus. Nat. Neurosci. 2, 266-270.

van Praag, H., Schinder, A. F., Christie, B. R., Toni, N., Palmer, T. D., and Gage, F. H. (2002). Functional neurogenesis in the adult hippocampus. Nature 415 , 1030-1034.

van Praag, H., Shubert, T., Zhao, C., and Gage, F. H. (2005). Exercise enhances learning and hippocampal neurogenesis in aged mice. J. Neurosci. 25, 8680-8685.

Wolf, S. A., Kronenberg, G., Lehmann, K., Blankenship, A., Overall, R., Staufenbiel,M., and Kempermann, G.
(2006). Cognitive and physical activity differently modulate disease progression in the Amyloid Precursor Protein (APP)-23 Model of Alzheimer's Disease. Biol. Psychiatry 60, 1314-1323.

Conflict of Interest Statement: The authors declare that the research was conducted in the absence of any commercial or financial relationships that could be construed as a potential conflict of interest.

Received: 28 August 2009; paper pending published: 21 September 2009; accepted: 09 October 2009; published online: 10 November 2009.
Citation: Fabel K, Wolf SA, Ehninger D, Babu H, Leal-Galicia P and Kempermann G (2009) Additive effects of physical exercise and environmental enrichment on adult hippocampal neurogenesis in mice. Front. Neurosci. 3:50. doi: 10.3389/neuro.22.002.2009

This article was submitted to Frontiers in Neurogenesis, a specialty of Frontiers in Neuroscience.

Copyright $\odot 2009$ Fabel, Wolf, Ehninger, Babu,Leal-Galicia and Kempermann. This is an open-access article subject to an exclusive license agreement between the authors and the Frontiers Research Foundation, which permits unrestricted use, distribution, and reproduction in any medium, provided the original authors and source are credited. 